\title{
Integration of planning systems and an agent-oriented MES
}

\author{
P. Verstraete, ${ }^{*}$ P. Valckenaers, \\ B. Saint Germain, H. Van Brussel and \\ K. Hadeli \\ Department of Mechanics, \\ Faculty of Applied Science, K.U. Leuven, Belgium \\ E-mail: paul.verstraete@mech.kuleuven.be \\ E-mail: paul.valckenaers@mech.kuleuven.be \\ E-mail: bart.saintgermain@mech.kuleuven.be \\ E-mail: hendrik.vanbrussel@mech.kuleuven.be \\ E-mail: hadeli.karuna@mech.kuleuven.be \\ ${ }^{*}$ Corresponding author
}

\begin{abstract}
Today, industry is confronted with a fast changing market. Customers demand a high-quality, low price and tailored product. This requires flexible manufacturing systems. At K.U. Leuven, a Manufacturing Execution System (MES) is designed, that allows to adjust execution to quickly changing circumstances. This paper discusses how this MES can cooperate with existing planning systems. A collaboration mechanism is presented and the advantages of this collaboration are discussed.
\end{abstract}

Keywords: Manufacturing Execution Systems (MESs); plannings systems; multi-agent systems.

Reference to this paper should be made as follows: Verstraete, P., Valckenaers, P., Germain, B.S., Van Brussel, H. and Karuna, H. (2006) 'Integration of planning systems and an agent-oriented MES', Int. J. Manufacturing Technology and Management, Vol. 8, Nos. 1/2/3, pp.159-174.

Biographical notes: Paul Verstraete is a candidate for the $\mathrm{PhD}$ degree in mechanical engineering. Since 2003, he is with the Mechanical Engineering department.

ir. Paul Valckenaers received his $\mathrm{PhD}$ degree in Mechanical Engineering in 1993. Since 1986, he is with the mechanical department.

Bart Saint Germain is a candidate for the $\mathrm{PhD}$ degree in mechanical engineering. Since 2002, he is with the mechanical engineering department.

Prof. Hendrik Van Brussel is a Full Professor in Mechatronics and Factory Automation in the Faculty of Engineering, K.U.Leuven, Belgium. He is a fellow of SME and IEEE. He is a member of the Royal Flemish Academy of Belgium for Sciences and Arts, former President of CIRP (International Institution for Production Engineering Research) and Foreign Member of the Royal Swedish Academy of Engineering Sciences.

Hadeli is a candidate for the $\mathrm{PhD}$ degree in Mechanical Engineering. Since 2000 , he is with the mechanical engineering department. 


\section{Introduction}

Companies, to remain competitive, need to answer customer demand in a more and more changing market. Traditional manufacturing control provides a good long-term production optimisation. These systems, however, do not provide the capability to quickly adapt to changes and evolution in market demand. At K.U. Leuven, a Manufacturing Execution System (MES) is designed, that allows to adjust execution to quickly changing circumstances. By integrating this MES with existing planning systems, the advantages of both systems, long-term optimisation and robustness to changes, are obtained.

This paper discusses how to achieve this integration. To deploy the MES in industry, it is important to know which type of planning systems exists and how they communicate with the MES. This is given in the first paragraph. Next, a short overview of the model and the basic functioning of the MES are described. In the third paragraph, possible mechanisms for collaboration are presented. In the fourth paragraph, the changes to the collaboration compared to the current situation are described. Next, this work is compared with other approaches. Finally, the conclusions are given.

\section{Planning systems}

To understand the requirement to deploy a novel type of MES in industry, we need to take a closer look at which type of planning systems exists today. Firstly, a general description of a planning system and the MES is given. Also, the collaboration between these two systems is given. Then, the different types of planning systems are elaborated. For each type, their input, functioning and output are explained. As a conclusion, an overview is given for different types of planning systems.

\subsection{General description}

A planning system is not just one tool. It consists of the subsystems intended for Customer Relationship Management, Human Resources management, planning, demand forecasting, inventory management and so on. In short, the combination of all these systems provides management with a means to plan and control its manufacturing activities and supporting operations.

An MES (Mesa International, XXXX) is a "manufacturing oriented system that interacts and shares information with other manufacturing applications. These are the information systems that reside on a plant floor between the planning systems in offices and direct industrial controls at the processes itself." MESs have five core tasks:

- tracing the execution on the shop floor

- supervising the process control systems

- supervising the routes that products follow

- supervising when and where operations on products start

- handling disturbances to the execution (failure of a machine, late or wrong delivery, etc.).

Systems today mainly perform the first task. 
The collaboration between the MES and the planning system occurs by releasing a short-term planning ( 4 or 5 days horizon) on a regular basis (a few times a day).

The rest of this section gives an overview on the different types of planning systems, used in the industry today. For every type to be indicated which kind of planning has to be delivered by the system.

\subsection{MRP systems}

MRP stands for 'Material Requirements Planning'. The first planning systems introduced in industry were of this type. Their performance is very slow. Typically, one run of the entire system could take a couple of days in the past, and still takes several hours today.

The functioning of the system is described as follows:

- Input: the MRP system requires an Master Production Schedule (MPS) as an input. An example of an MPS is given in Table 1. The most important row in this table is the MPS row. It states the production levels for the long term (3-4 months), for each week, for the entire company. Only a small part is included in the example. A more detailed discussion of the MPS can be found by Sipper (1998).

- Functioning: of the entries mentioned in the MPS, an explosion of the bill of materials is performed. In this process, fixed lead times were used. Usually, these lead times overestimate the real lead time severely, to compensate for the possible disturbances or insufficient capacity.

- Output: the output consists of three main parts:

- the MRP list, shows details of deliveries from suppliers and to clients

- the order list, used for issuing materials and production orders

- the exception list, indicates missed due dates and late deliveries.

In this output, the order list is considered as the planning for this system.

Table 1 An example of MPS

\begin{tabular}{lrrr}
\hline \multirow{2}{*}{ Current inventory $=1600$} & \multicolumn{3}{c}{ Week } \\
\cline { 2 - 4 } & 1 & \multicolumn{1}{c}{2} \\
\hline Forecast & 1000 & 1000 & 1000 \\
Customer orders to deliver & 1200 & 800 & 300 \\
Inventory at the end of the week & 400 & 1900 & 900 \\
MPS & 0 & 2500 & 0 \\
\hline
\end{tabular}

\subsection{MRP II systems}

MRP II systems is a further improvement over the MRP systems. After the MRP runs, the process goes to several further stages. This is called a control hierarchy.

- Input: the input is still the MPS, as for the original MRP system 
- Functioning: MRP II systems add more steps to this hierarchy:

- Capacity planning: this occurs at a low level of detail. Because of the fixed lead times, this planning is very rough and vulnerable to variation in processes.

- Shop floor control: it provides a more detailed planning in a short term. The market for this type of planning is very dispersed. A lot of these systems are made in-house. In other words, the needed critical user mass for developing mature software is lacking. Because they have to respect the rough allocations already made, their planning too is vulnerable to variation.

- Output: the output is a list of orders, with allocations to resources. Because of the variability in manufacturing processes, the allocations made in the planning are typically invalidated within an hour.

\subsection{ERP systems}

ERP stands for 'Enterprise Resource Planning'. ERP systems integrates the MRP II system with other functional areas within a company. It provides an enterprise-wide database, operating on a common computing platform, interacts with an integrated set of applications and consolidating all business operations in a single IT environment. Ideally, an ERP system allows information to be entered only once.

The functioning of the ERP system, from a planning point of view is not different from that of an MRP II system.

\subsection{SCM systems}

SCM systems stand for 'Supply Chain Management' systems. These systems resolve some shortcomings of the ERP systems. They plan and optimise the supply chain by simultaneously considering demand, capacity and material constraints. The main techniques used are linear programming, constraint logic programming and simulation.

SCM systems differ mainly from the previous systems, in their inner functioning. The output of this system to the execution systems is not different from that of an MRP II system: a list of orders, with mentioning the time orders of which needs to be executed and the machine where execution needs to be performed.

SCM systems cover less areas than ERP systems. By using more efficient algorithms and being less integrated, SCM systems are generally considered faster than ERP systems. However, for collaboration with HR, CRM, ... systems, integration with an existing ERP system is needed. Two main trends can be seen:

- SCM solutions provided by ERP vendors: major ERP vendors have noted the disadvantages of their systems, and are developing SCM systems of their own. A typical example is SAP SCM (XXXX). Integration of this system with the SAP R3 ERP system is of course excellent. Other players are PeopleSoft, Oracle, etc.

- Integration of best of breed SCM solution with ERP system: currently, important players in the SCM market are I2 Technologies (XXXX) and Manugistics (XXXX). For collaboration with the major ERP packages they have to rely on integration software. A typical example is I2 Rhythm. 


\subsection{Conclusion}

This section discusses the different types of planning systems, and their communication with the execution system was studied. Table 2 gives an overview of the different types of output of the planning systems. All systems specify which order needs to be executed for a given period. The response time varies over the different systems. Also, the resources on which the planning should be executed are not always specified. As MRP systems are still used in a lot of companies today, the chance that the MES has to function without this information being present is considerable.

Table 2 Comparison between the output of the different planning systems

\begin{tabular}{lll}
\hline Planning system & Output to execution system & Response time of planning system \\
\hline MRP & List of orders & Several hours \\
MRP II & $\begin{array}{l}\text { List of orders } \\
\text { assigned to resources } \\
\text { with start and stop time }\end{array}$ & Several hours \\
& $\begin{array}{l}\text { List of orders } \\
\text { assigned to resources } \\
\text { ERP }\end{array}$ & Several hours \\
& $\begin{array}{l}\text { Lith of orders and stop time } \\
\text { assigned to resources } \\
\text { with start and stop time }\end{array}$ & \\
SCM & & \\
& & \\
\end{tabular}

\section{K.U. Leuven PMA MES}

This section discusses the multi-agent MES developed by K.U. Leuven-PMA. An in-depth discussion is not in the scope of this paper, and can be read by Valckenaers and Van Brussel (2005) and Wyns (1999). Firstly, the model on which this MES operates is discussed. Secondly, the basic operation of the MES is presented. Finally, an example scenario is discussed.

\subsection{The model of the MES}

The model used for this execution system is the shop floor itself. Every element in the shop floor is represented in the execution system as an agent, as illustrated in Figure 1. The rest of this paragraph discusses the basic types of agents present in this system, following the PROSA reference architecture (Wyns, 1999).

- The operators, machines, tools, . . in the system are represented as agents, more specifically resource agents. These agents continuously refresh the status of the resource they represent. For example, if there is a breakdown, the agent will be capable of providing this information to other agents almost immediately. This agent has deep and detailed knowledge about the resource. This knowledge enables the resource agent to give information to other entities in the system about his performance under given conditions. It can be compared to a reservation department in a hotel. When asked, a hotel can give information about availability of the rooms, for which period and at what price. These agents have a goal to maximise the service delivery of their machine (includes usage maximisation if heavily loaded), under given conditions. 
- The orders of clients, as well as production orders are represented as order agents. These agents have the latest information about the part(s) that are involved in the order and the client that made the order (if present). These agents have a goal to get their order produced correctly and on time.

- The process and product knowledge for producing an order is represented as product agent. This knowledge can be compared to the recipe of a baker to bake a cake. This agent keeps informed of the latest technical innovations and is capable of sharing this knowledge with other agents.

Figure 1 Reality as the model of the MES

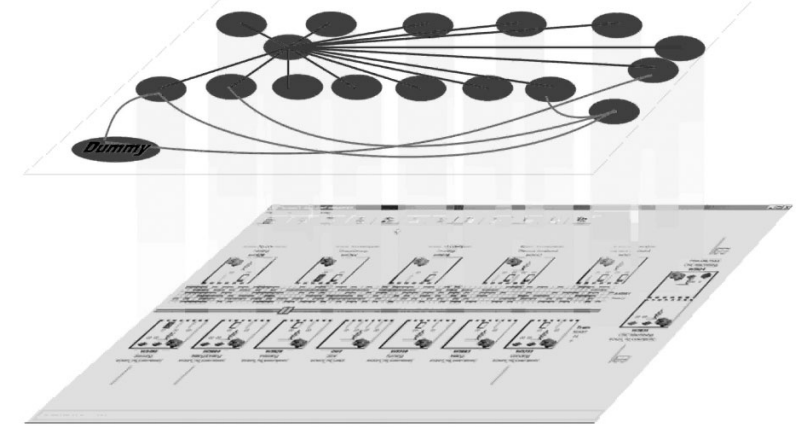

All these agents have the latest information about the element they represent in reality. By refreshing this information on a regular basis, every element in the manufacturing system is capable of obtaining a view on the manufacturing system, that is consistent with reality. How this view is obtained, to be discussed in the next section. The agent can obtain this information, without being exposed to the complexity of the entire system. Indeed, a customer of a hotel does not need to know how a hotel works, to be capable of making a reservation.

\subsection{The basic operation of the multi-agent MES}

The software system allows the order agents to search for interesting solutions. Order agents send out scouts, called exploring ants. ${ }^{1}$ Such a scout tries to discover one possible way of travel over the virtual factory, formed by machine-agents and the connections between them. This exploring activity can be compared to the preparation of the booking of a holiday trip. At each node, the scout enquires at each resource agent how much time a reservation would cost, what the quality of the operation would be, ... The results of this journey are reported back to the order agent.

The software system makes it possible for an order to reserve a given resource. These reservations are spread by another type of ant agent, an intention ant, over the virtual factory. Reservations disappear if they are not reconfirmed regularly. This approach makes an explicit cancellation policy unnecessary.

The software system gives a forecast to the worker on the shop floor of the intentions of the other worker. The worker can access this information via the resource agent that represents 
him. These forecasts are the reservations made by the intention ants. By providing the capabilities described above, the production control system can provide short-term forecasts of future reservations on every node in the shop floor.

These short-term forecasts can only be reliable if the order agents follow their intentions as a rule. To ensure this, the software system imposes socially acceptable behaviour upon the members of the agent society.

More specifically, order agents can only change intentions if the perceived amelioration is significant. The threshold for changing a job in the near future is higher than for changing a job in a more distant future. This can be compared to the way by which meetings are planned. Suppose a person cannot join the meeting and later warns the other participants, the bigger the inconvenience he creates. Therefore, a better justification for this change will be expected (e.g. sickness).

Secondly, the order agents change their intentions probabilistically. This is given in the following section.

\subsection{Example scenario: a machine failure}

In this section, an example scenario is discussed to illustrate the operation of the multi-agent MES. Firstly, the machines and orders present on the shop floor are described. Secondly, the exploration and reservation of the orders are described. Finally, we consider a breakdown of a machine, and see how the system adjusts to the situation.

Consider a factory topology with four machines, M1, M2, M3 and M4. M1 and M2 support one operation, O1; M3 and M4 support another one, O2. Both lines, M1-M2 and M3-M4 have equal processing time. There are two orders in the system, order 1 and order 2. Note that the system does not suppose the order or present machines to be known beforehand. All these things are discovered at run time.

M1, M2, M3 and M4 are represented in the multi-agent system by four resource agents. Each resource agent knows the other resource agents connected to him. M1 knows M2 is connected and M3 knows M4 is connected. The entrance knows M1 and M2 can be reached from there. The different agents also monitor these connections. If they change, they will notice this fact.

When the two orders enter the system, order agents are launched. Both orders send out scouts. This is the situation in phase 1 in Figure 2(a).

These scouts, send out by the order agents, report back equal executions times. At a certain point, the first order reserves the first line, M1-M2 (line 1). After a certain time, the scouts of order 2 report worse performance for line 1 . This causes order 2 to reserve the second line, M3-M4. This is illustrated in phase 2 in Figure 2(b).

A breakdown on M2 occurs. This is represented as if a reservation of the highest priority would have been made for the estimated repair time on M2. This deteriorates the performance of the line M1-M2. This is reported to order 1 by his scouts. Depending on the estimated repair time, the order will switch to the other line, line 2, with a high probability (phase 3, Figure 2c).

Note that the order does switch intentions probabilistically. This avoids chaotic behaviour in the system. If all orders in the system would switch intentions immediately if they perceive the breakdown, this could lead to a queue being built up in front of line M3-M4. Line M1M2, in turn, would become the most attractive line. This in turn would cause another switch, which would make line M3-M4 the most attractive, and so on. An unstable system would be the result. By switching probabilistically, some order agents detect that waiting for repair (of M2 in this case) has become their best option, and leave their intention unchanged. 
Figure 2 A machine failure
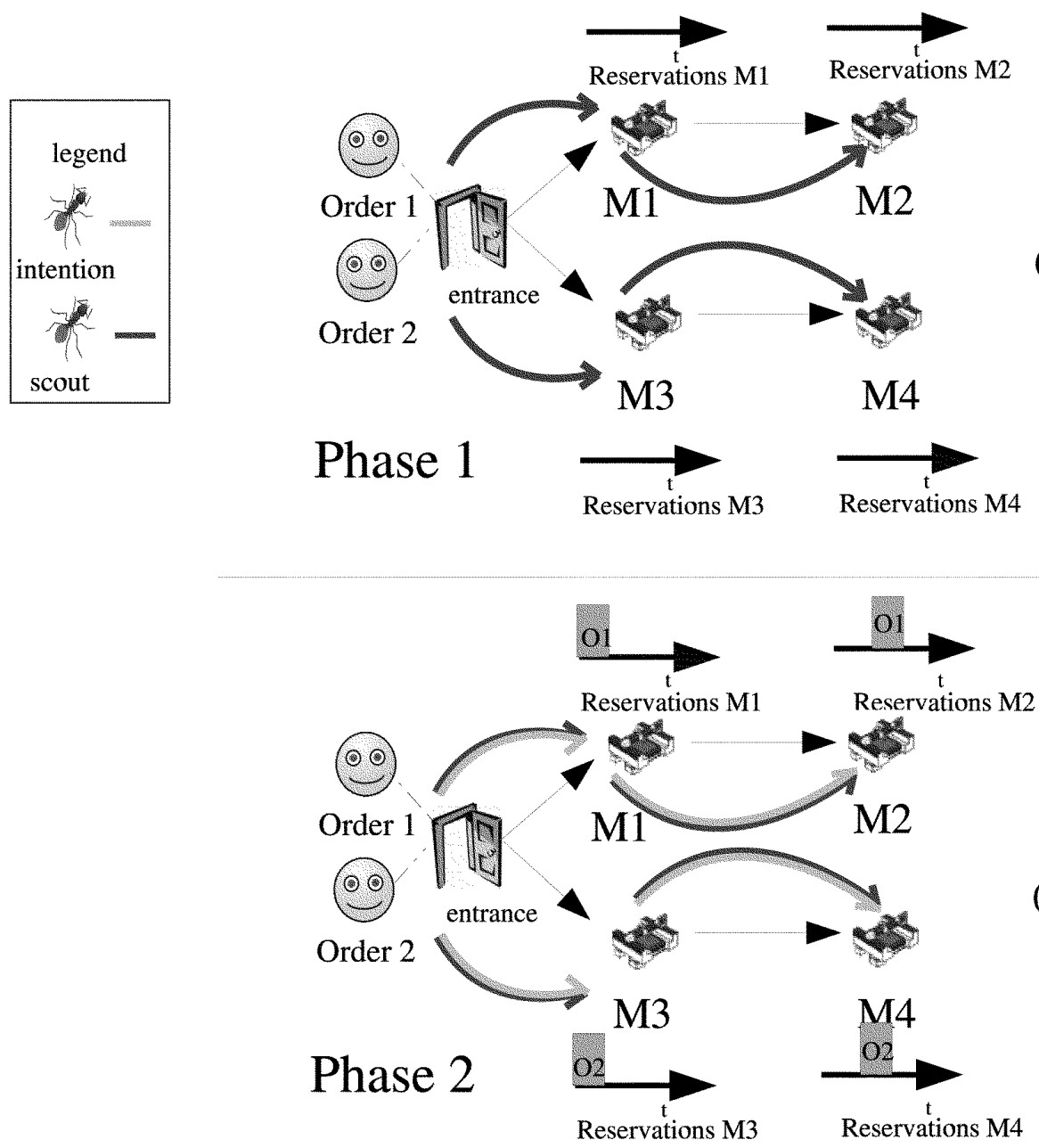

(b)

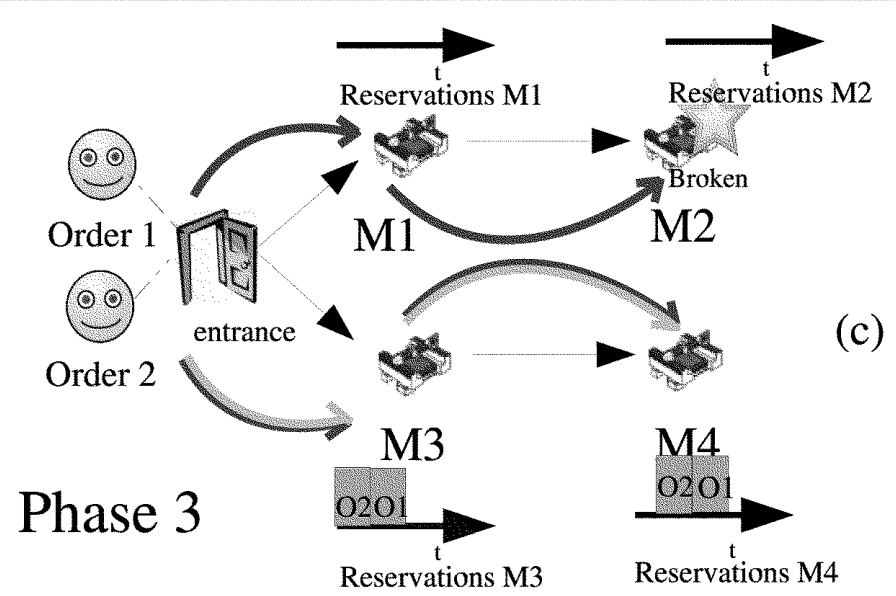


The exploring ants travel at intervals of a few seconds or less over the topology. Because of the slower rate by which changes occur on the shop floor (minutes), several exploring rounds can be performed before taking a decision. This allows the order to evaluate different alternatives.

\subsection{Conclusion}

In this section, the model and the basic functioning of the MES are described concisely. This functioning is illustrated with a scenario, the failure of a machine. Note that no exception handling is needed to handle the disturbance.

\section{Collaboration}

In this section, several ways to deploy the MES in the industry are explored. Firstly, the assumptions the MES can(not) make on the planning system are described. Secondly, a basic collaboration mechanism are described. Thirdly, aggregation and the use for collaboration are presented. Finally, the conclusions are given.

\subsection{The assumption the MES can make}

- List of orders: every planning system described, at least gives a list of orders that need to be performed in the near future (e.g. the next week).

- Resource allocation and start time of execution: MRP systems just provide a list of orders, no resource allocation or start or ending time of the operation. Therefore, the system should not crash if this information is not present. If this information is present, the MES should be capable of using it, however.

- Response time: only SCM systems can respond to a disturbance in a matter of minutes. Therefore, there should certainly not be an assumption that his capability is present. Rescheduling too frequently also is not favourable. This leads to instable and frequently changing plannings, which makes the execution more difficult.

\subsection{A basic collaboration scheme}

This basic scheme comprehends the following issues: if a scenario is present in the planning:

- for every job in the planning, an order agent is created.

- a significant fraction of the scouts, sent out by these order agents, explore the scenario in the planning (resource allocations and timing). Another fraction still operates like before and searches a favourable route independently.

- the order agents evaluate how well the reports brought back by the exploring ants, meet the requirements in the planning. Adherence to these requirements increases the chance of selection.

- the order agent considers the scenario that is present in the planning as his original intention. An agent has a resistance to changing his planning. Therefore, an order agent will not deviate from the scenario in the planning, unless this has severe consequences (e.g. unable to meet the requirements).

If no scenario is present, the order agent operates as described in the previous sections. 
Note that this basic scheme allows a planning to be included in the system. This is used without any modification on the basic operation of the multi-agent system.

\subsection{The use of aggregation}

This section first indicates the need for aggregation. When aggregation is defined, after which an example is given on how it can be used in the collaboration with planning systems.

The planning introduces several requirements. Examples of these requirements are the completion of the jobs, the time by which these jobs need to be completed, the quality of the finished goods, ... These requirements influence the possibilities an agent has in the system. As much requirements as possible should be fulfilled by the intentions of the agents, on short and long term.

These requirements address more than one agent. For example, it is not desirable for one order to meet the deadline in the planning, if all other orders fail to meet their deadline due to this fact. Therefore, aggregated agents should be used.

Aggregated agents (Wyns, 1999) are defined as a set of related agents that are clustered together and form on their turn a bigger agent. One agent can be a part of several aggregated agents. An aggregated agent can be a part of a bigger whole.

In Figure 3, a planning can be represented as an aggregated agent. It consists of several order agents. The aggregated agent translates the requirements of the planning to requirements to the order agent. For example, the planning agent gives due dates to every order agent involved.

Suppose an order agent claims a resource and prevents all other orders to meet their due dates, there are two possibilities.

- the planning agent observes this independently, and intervenes

- an order agent is frustrated by not being able to meet his requirements. He alerts the planning agent of this fact.

In both cases, the planning agent can intervene by giving the solution of the greedy order agent a very bad reward. This causes the order agent to choose another option.

A simple example is given now. Three jobs need to be completed in a restaurant before customers arrive. The food is to be delivered in the morning. The faster this food can be processed and be put into cooling installations the better. Suppose the planning consists of three orders that need to be executed. There is one place in the restaurant where the food can be processed. One job needs $50 \mathrm{~min}$ to complete. The other two jobs only need $10 \mathrm{~min}$.

For each job an order agent is created. The planning, consisting of the three jobs, is represented as an aggregated agent. This agent knows his part, the three order agents, and each order agent knows the aggregated agent. The goal of the planning agent is to deliver each order in one day and without leaving the food too long outside the cooling system. The time each order type may reside in the restaurant before it should be cooled, can be seen in Figure 4.

Each order agent sends out scouts. The scouts discover the place where the meat can be processed and report back to the order agent. Suppose the job that takes $5 \mathrm{~h}$ is the first one to reserve the restaurant. The others reserve the restaurant after that. The resulting planning is shown in Figure 4. 
Figure 3 A planning represented by an aggregated agent

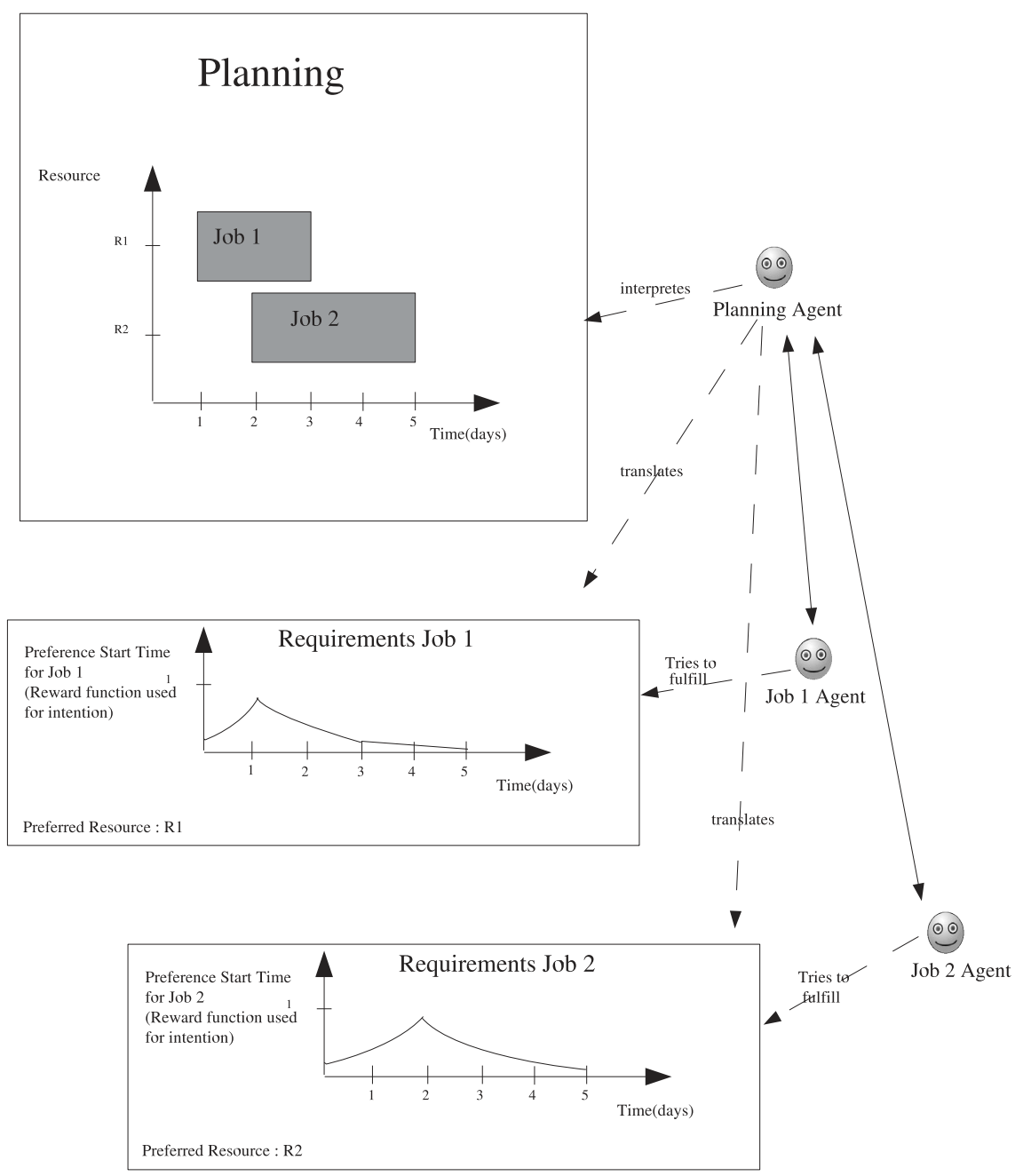

This planning is not acceptable for both jobs 2 and 3. These jobs alert the aggregated order of this fact. This aggregated order now exercises pressure on the first job to release his slot. This is illustrated in Figure 5. As a result of this, the first job releases his slot after some time. This gives the two other jobs the chance to get a better slot. In the final planning, all orders are satisfied (see Figure 6).

\subsection{Conclusion}

This section discussed two collaboration schemes. Both schemes succeed at introducing the planning into the multi-agent system. This makes collaboration with existing planning systems possible. Note that neither of the two schemes require modifications to the basic operation of the multi-agent MES. 
Figure 4 Restaurant example: the job that takes $50 \mathrm{~min}$ is the first one to reserve the food processing department

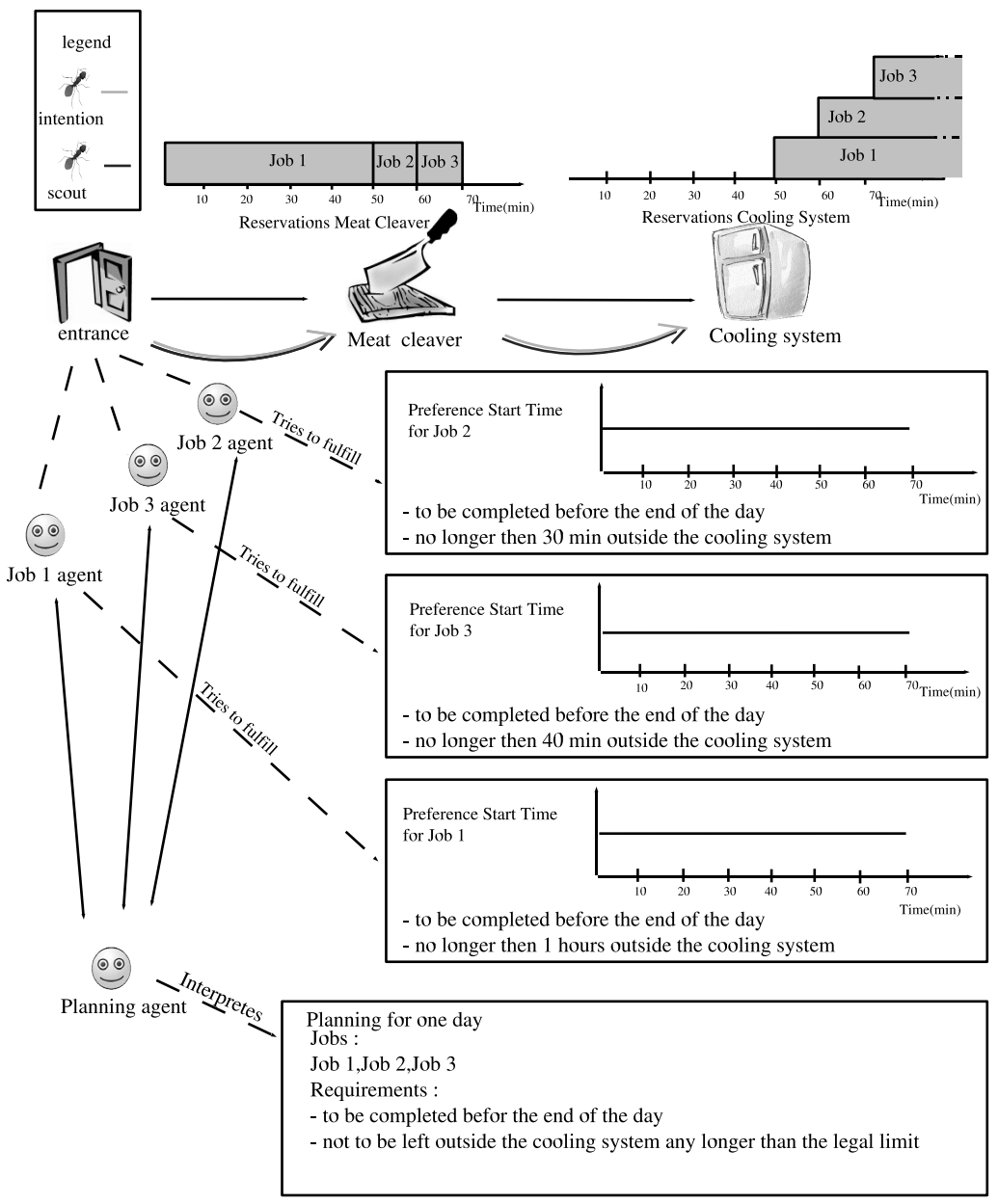

\section{Advantages of the collaboration}

In this section, the changes to the collaboration by deploying the multi-agent MES are discussed. An overview is given in Table 3.

Table 3 Comparison between collaboration before and after the deployment of the MES

\begin{tabular}{lll}
\hline Criterion & Before deployment & After deployment \\
\hline Requirements & $\begin{array}{l}\text { Adding requirements only } \\
\text { possible when generating planning }\end{array}$ & $\begin{array}{l}\text { Adding requirements also } \\
\text { supported by MES }\end{array}$ \\
Disturbances & $\begin{array}{l}\text { Handled by planning system } \\
\text { exception handling }\end{array}$ & $\begin{array}{l}\text { Handled by MES } \\
\text { business as usual }\end{array}$ \\
& & \\
\hline
\end{tabular}


Figure 5 Restaurant example: the aggregated order agent perfoms pressure on the first job

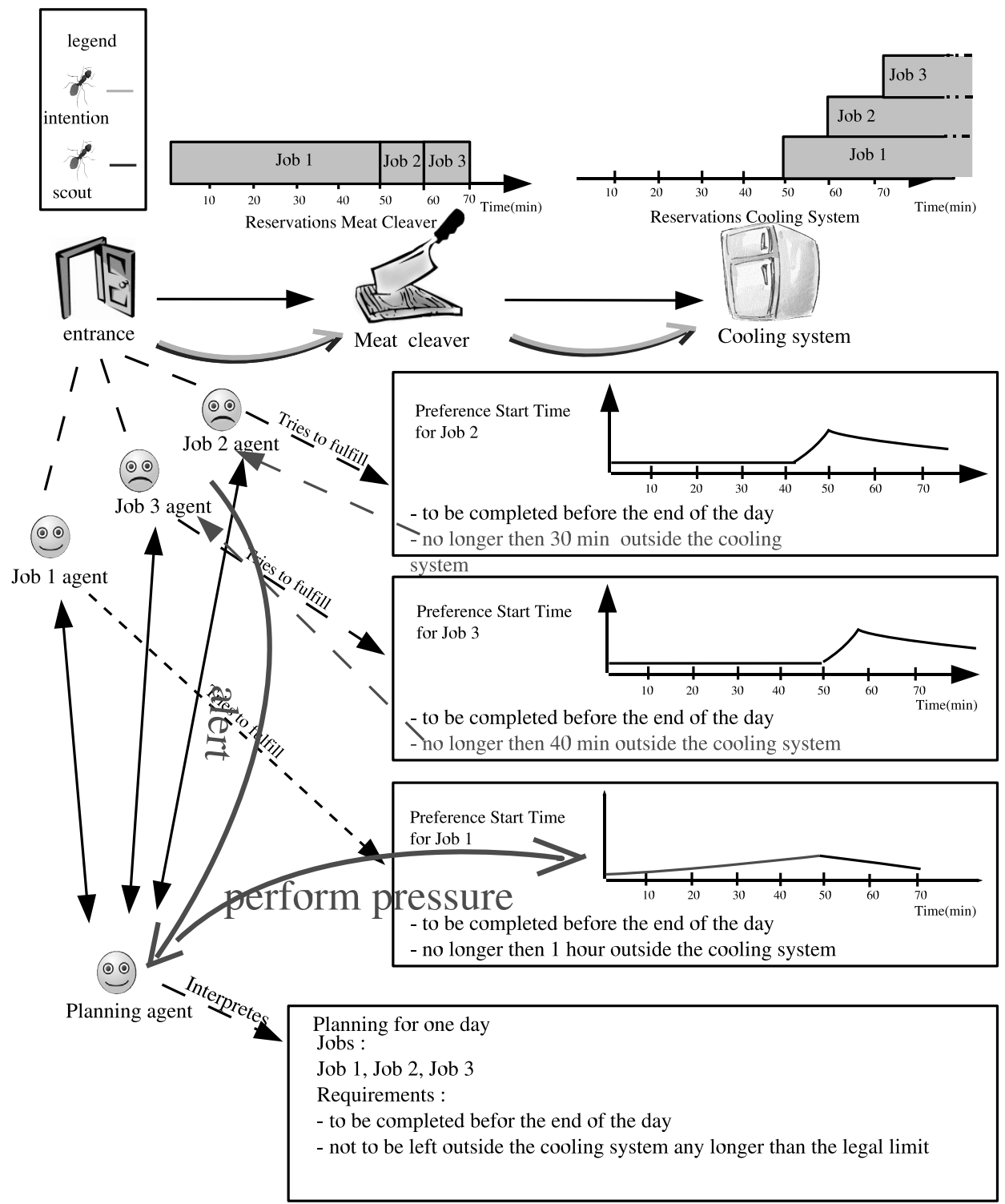

\subsection{Handling of disturbances}

As indicated before, SCM systems are capable of taking new constraints, and regenerate a planning in a few minutes. However, frequent replanning leads to an instable situation. Other entities, suppliers and clients base their intentions on the planning generated. A quickly changing could be plan, even when respecting hard constraints, is a weak basis to base decisions on.

After introducing the multi-agent MES, commitments to other entities (clients and suppliers) are a fundamental mechanism in the system. Because of the social behaviour 
of the agents (stick to intention if possible), they will search to solve disturbances, without minimal disturbance to the commitments to other agents. This allows the planning system to reduce its replanning rate.

Figure 6 Restaurant example: the resulting planning

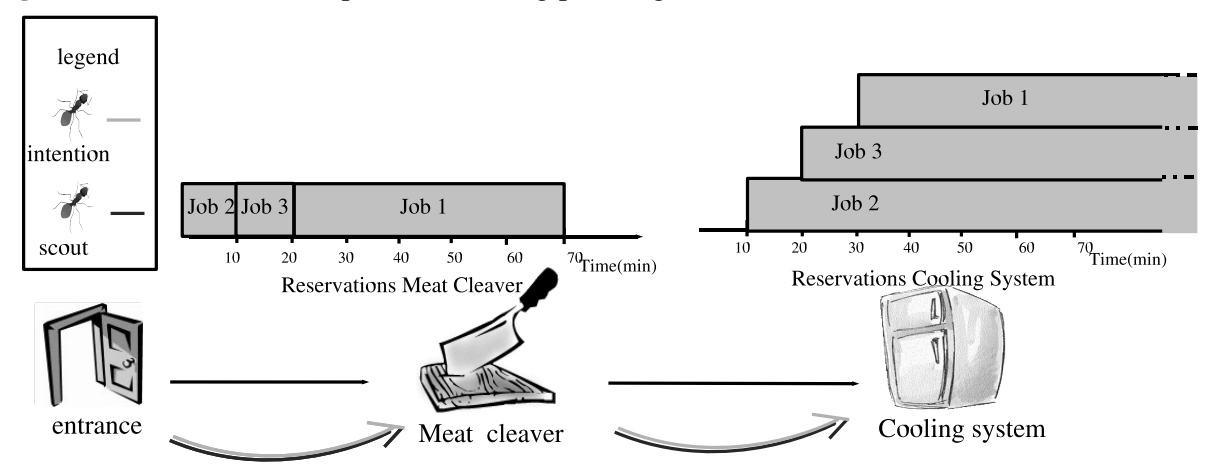

\subsection{Adding requirements}

When generating a planning, a goal is pursued. This goal depends on the dynamic situation on the shop floor. In the example of the restaurant, the goal is to produce all jobs in time, without wasting the food by leaving it too long outside the cooling system. One could imagine the cook falling sick, and only its assistant being left to process the food. This assistant might not have the experience to process certain jobs. So, by this disturbance, the experience available has decreased. It is now the goal to meet as much of the former requirements as possible, with the given skills available. Another example is kosher food. As a result the food should not be processed together with porch. This should also be included when evaluating the found solutions.

Before the multi-agent MES was deployed, is necessary to model all possible requirements in the goal function of the planning system.

- If not, they could not be taken into account when evaluating a solution.

As shown by previous examples, the health of a cook or specific client can become important under certain circumstances. A data model is always designed with the most frequent constraints in mind, for example, capacity constraints. Adding constraints, not anticipated at design time, is very difficult in most cases.

- The manufacturing problem is not tractable (Parunak, 1991). Therefore, not all constraints can be taken into account when calculating the solution. Some constraints need to be simplified. This leads to unrealistic assumptions of the planning system about the reality. For example, the health of a cook is disregarded because of the number of constraints that needs to be considered already (capacity constraint, time constraints, etc.).

- The people on the shop floor have to resolve unrealistic assumptions in the planning without support. They form 'the control system' on operational level. Existing software systems on this level are merely used for tracing the execution. 
After deploying the MES, requirements can still be added after generating the planning:

- There is no need for explicit modelling of the goal function. Each agent pursues his own goal. By coördinating with different agents, a global solution emerges.

- Minimal exposure of agent to internal functioning of other agents. For example, suppose, when generating the planning, only one type of order agent is known. These order agents just want to produce their job in time, with an acceptable quality. When executing, it turns out that some orders are meant for kosher food (no contact with porch meat). The type of these orders is modified. This new type demands to be processed on resources, where no porch has been processed. These orders scout for possible solutions, and make reservations, as before. No adjustments to the rest of the system need to be made to make this possible.

- Only local knowledge is needed for an agent to be capable of functioning. Indeed, a resource agent only needs knowledge of its own processes, but does not need to take any routing decisions. Likewise, an order agent does not need any process knowledge. Therefore, every agent is only confronted with a small part of the problem, which remains tractable.

In the MES, the agent is enriched by the experience of the people on the shop floor. For every worker on the shop floor, a resource agent exists in the agent system. This is also true for every job in the system. Therefore, it is easy for a person on the floor, to detect which part of the control system corresponds to his responsibility. He can influence this agent, without having detailed knowledge on the rest of the system.

\section{Related work}

This work builds further on achievements made in the holonic community. This section presents two alternative holonic approaches. It discusses the difference between these two approaches and the approach presented here.

The ADAptive holonic COntrol aRchitecture (ADACOR) (Leitao, 2004), for distributed manufacturing systems, aims to balance dynamically between a more centralised structure and a more a decentralised one. This balancing is achieved by explicitly modelling the stationary state and the transient state. The stationary state is active when there are no disturbances, the transient state when there are. Propagation of pheromones is only used if disturbances occur and a transition of the one state to the other is needed. In this approach, the basic agents of the K.U. Leuven PMA MES use the propagation of pheromones as a coordination mechanism. As explained in the previous section, there is no explicit handling mechanism present, exceptions are 'business as usual'.

Bongaerts (1998) developed PHOCS, an MES and PharSIFal, a planning system, as an example of how an MES and a planning system could cooperate. In this example; however, PHOCS was explicitly designed for cooperation with PharSIFal. In the approach presented here, the MES should not rely on the presence of the planning system. No adaptations to design principles and mechanisms should be made regardless of the operation mode.

\section{Conclusion}

This paper began by examining the different types of planning systems. All systems provide the execution with a planning on a regular basis. These systems differ in the time they need to recalculate the planning and the information they deliver to the execution systems. 
A collaboration mechanism is presented, allowing the manufacturing execution system, developed by K.U. Leuven, to collaborate with the different types of planning systems. This mechanism does not require modifications to the basic functioning of the MES.

This collaboration mechanism brings two main changes to the current collaboration:

- there is no longer a need to specify all requirements in a central way. If a requirement becomes known after the generation of the planning, it is taken into account by the MES

- disturbances can be resolved by the MES. This reduces the required replanning rate, which in turn makes the planning more stable.

\section{Acknowledgements}

This paper provides results obtained through the work sponsored by the K.U. Leuven Concerted Research Action $\left(\mathrm{GOA}-\mathrm{AgCo}_{2}\right)$. The authors assume the scientific responsibility.

\section{References}

Bongaerts, L. (1998) 'Integration of scheduling and control in holonic manufacturing systems', Doctoral Thesis, K.U. Leuven, Available at: http://users.pandora.be/luc.bongaerts/doc/ LucBongaertsPhd.html.

Indriyadi, Y. (2002) 'Distributed dispatching control for dynamic flow-line manufacturing systems', Doctoral Thesis, K.U. Leuven.

I2 Technologies (2005) Available at: http://www.i2.com.

Leitao, P. (2004) 'An agile and adaptive architecture for manufacturing control', Doctoral Thesis, University of Porto, Available at: http://www.ipb.pt/pleitao/papers/pjl-tese.pdf.

Manugistics (2005) Available at: http://www.manugistics.com/solutions/SupplyManagement/ cg_sn.aspx?id=SupplyManagement.

Mesa International (2005) Available at: http://www.mesa.org.

Parunak, H. (1991) 'Characterizing the manufacturing scheduling problem', Journal of Manufacturing Systems, Vol. 10, No. 3, pp.241-259.

SAP SCM (2005) 'Technical Details' Available at: http://www.sap.com/solutions/business-suite/ $\mathrm{scm} /$ technicaldetails/index.aspx.

Sipper, D. (1998) Production: Planning, Control and Integration, McGraw-Hill.

Valckenaers, P. and Van Brussel, H. (2005) 'Holonic manufacturing execution systems', CIRP AnnalsManufacturing Technology, Vol. 54, No. 1, pp.427-432.

Wyns, J. (1999) 'Reference architecture for holonic manufacturing systems - the key to support evolution and reconfiguration', Doctoral Thesis, K.U. Leuven, Available at: http://people. mech.kuleuven.ac.be/jwyns/phd/order.html.

\section{Note}

${ }^{1}$ Design inspired by ant colonies, hence this terminology. 